Claremont Colleges

Scholarship@ Claremont

All HMC Faculty Publications and Research

HMC Faculty Scholarship

$1-1-1986$

\title{
Chemical Applications of Scanning Tunneling Microscopy
}

Paul West

John Kramer

David V. Baxter

California Institute of Technology

Robert J. Cave

Harvey Mudd College

John D. Baldeschwieler

California Institute of Technology

\section{Recommended Citation}

West, P.; Kramer, J.; Baxter, D.V.; Cave, R.J.; Baldeschwieler, J.D. “Chemical Applications of Scanning Tunneling Microscopy," IBM Journal of Research and Development 1986, 30, 484.

This Article is brought to you for free and open access by the HMC Faculty Scholarship at Scholarship @ Claremont. It has been accepted for inclusion in All HMC Faculty Publications and Research by an authorized administrator of Scholarship @ Claremont. For more information, please contact scholarship@cuc.claremont.edu. 


\section{Chemical applications of scanning tunneling microscopy}

by Paul West

John Kramar

David V. Baxter

Robert J. Cave

John D. Baldeschwieler structure of molecular species including reactants, products, and some of the reaction intermediates.

\section{Project status}

- Instrumentation

In order to achieve vacuum tunneling, a controllable vacuum gap must be maintained. Herein lie the major experimental constraints and difficulties of the STM. Mechanical isolation must be achieved which will allow extraneous vibrations to perturb the distance between the tip and the surface no more than $0.05 \AA$ (to achieve $0.1 \AA$ vertical resolution). Also, a method of controlling this distance and positioning the tip in the surface plane must be provided. For the highest resolution, the system must also be ultrahigh-vacuum (UHV) compatible.

The Caltech system uses a Varian FC-112-based vacuu system which includes a 200-liter-per-second triode ion pump and an auxiliary titanium sublimation pump. We employ the spring isolation system which was originated at IBM $[4,5]$. The probe tip is positioned relative to the surface in two stages. For fine positioning, the tip is mounted on a piezoceramic assembly which can move the tip in the Cartesian coordinates. The fine-positioning assembly consis of two separate ceramics, one for surface plane $(x$ and $y)$ motion and another for surface normal $(z)$ motion. For coarse motion, the fine-positioning assembly is mounted on a piezoceramic "louse." The sample to be studied is mounted on a macor block in front of the probing tip. The STM probing tips have been made by grinding $1-\mathrm{mm}$ tungsten wire to a point. This gives an overall tip radius of rough grinding process $[4]$

Experimental result

There are several modes of operation of the STM with whic we have experimented. The simplest mode of operation is to study the dependence of tunneling current on the distance between the probe and the surface. This is done by maintaining a constant potential across the tunneling junction and decreasing the tunneling gap while monitoring the current. Plots of the logarithm of the current vs. the tipto-surface distance (as computed from the piezoelectric coefficients of the ceramic, and the applied voltage) yield straight lines, as predicted from a simple one-dimensional square-bartier, stationary-state calculation. From the same alculation, the slope of the line should be equal to a fundamental constant times the square root of the work function. This is borne out in our data for a scan of this type using a tungsten tip $(\phi=4.6)$ and a gold surface $(\phi=5.1)$ which gives a work function of 5.2 , which is reasonable considering the unprepared nature of the surfaces (Figure One can envision a study of the change in work function of a surface as a function of the identity and surface coverage of adsorbates. about a micrometer, but smaller mini-tips are created by the

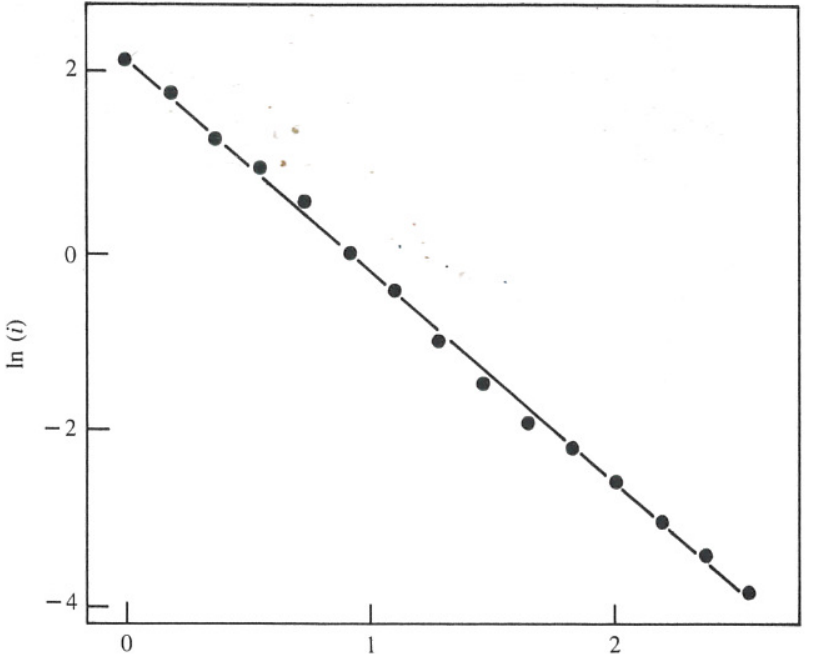

Figure 1 surface. The work function determined from the slope of the line is
s.

somewhat more complex mode of operation is that use to study the voltage dependence of current at constan distance. In this experiment we first stabilize the position of the tip at a fixed distance from the surface by running the constant-current feedback at a given initial voltage. Initial voltages are typically $10 \mathrm{mV}$, and the distance is adjusted to maintain a constant current of $5 \mathrm{nA}$, which corresponds 5-10 A. The feedback loop is then disconnected, and the voltage is quickly ramped in a triangular pulse using a PAR potentiostat. The voltage pulse and the current response as amplified by the Keithley electrometer are both stored versu time on a Tektronix Model 5223 storage oscilloscope. Figure 2 is a typical scan obtained by this mode of operation. The underlying structure observed in the current vs. voltage scan can be qualitatively explained in terms of a one-dimensiona square barrier distorted by an applied voltage [12]. Three-dimensional surface scanning is the third mode of operation with which we have experimented. In this case, a constant potential (typically $10 \mathrm{mV}$ ) is applied across the tunneling junction. The distance between the probe and the surface is adjusted by means of the feedback loop, which maintains a constant tunneling current of typically $5 \mathrm{nA}$. The probe is moved across the surface by changing the potentials on the $x$ and $y$ piezoceramics, and the voltage applied to the $z$ ceramic (as set by the feedback loop) is recorded as a function of $x$ and $y$ position.

Figure 3 is an example of surface scanning performed with our STM. The tip was a $1-\mathrm{mm}$ tungsten wire ground to a suited for the study of chemical processes on surfaces $[10,11]$. The orientation of the molecules with respect to 


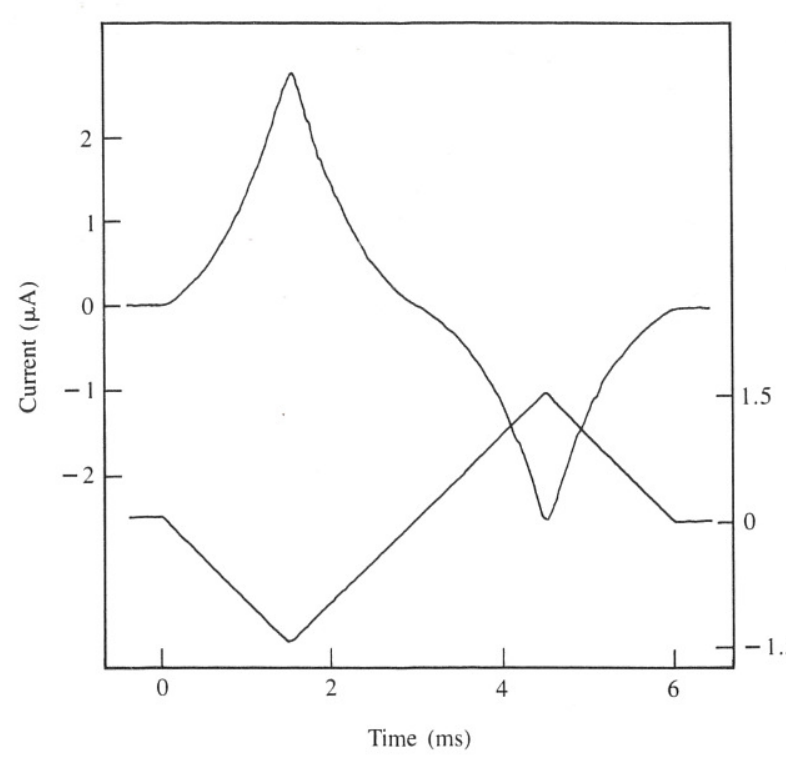

Figure

Plot of voltage applied across the tunneling gap and current observed vs. time at constant distance.

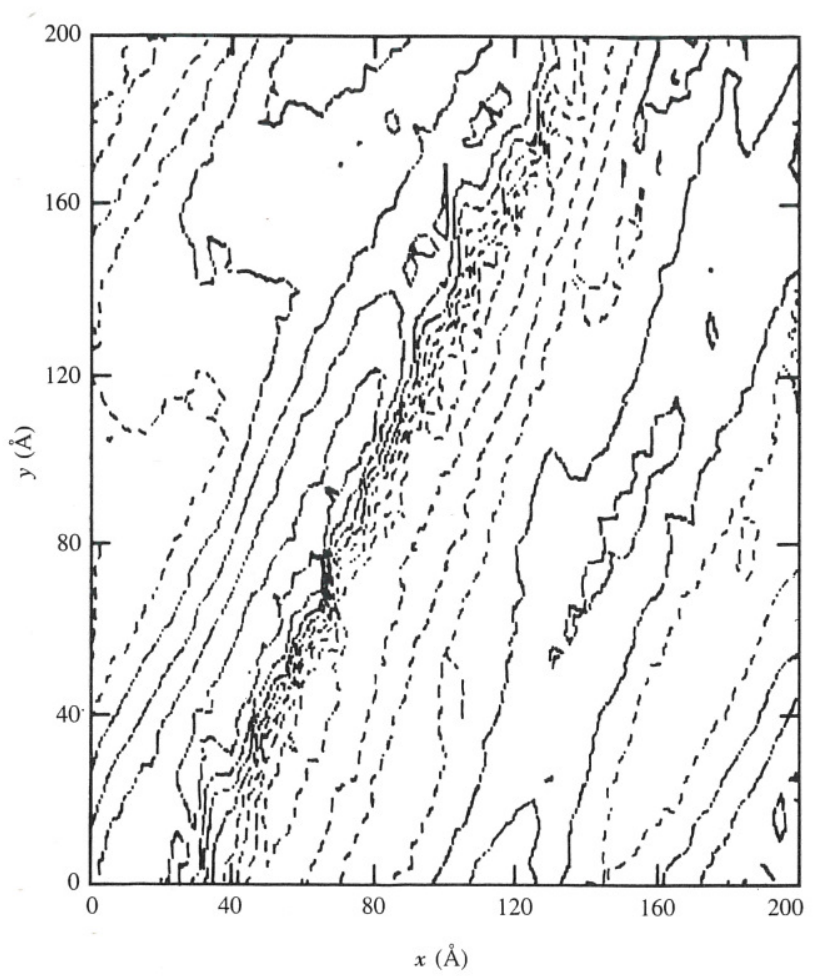

Figure 3

Contour plot of an STM scan of a 1000-Å thickness of gold vapor deposited on a glass slide. Solid lines represent positive contours, dotted lines negative contours. The vert
highest and lowest contour is about $160 \AA$ gold vapor $\sim 1000 \AA$ thick deposited on a glass slide. The together five passes over the surface. The origin of the

$\sim 160-\AA$ step which runs through the center of the scan is unknown, although it is similar to early results reported by other groups for uncharacterized gold surfaces [13]. A

subsequent scan on the $40 \times 40-\AA ̊$ square nearest the labeled origin is shown in Figure 4. In this scan, part of the $160-\AA$ step is also visible, indicating reproducibility of the scan.

\section{Future studies}

\section{Experimental objectives}

There is still much to be done before the potential of the scanning tunneling microscope in studying chemical processes on surfaces can be fully realized.

Recently a new type of vibration isolation system has bee developed by the researchers at IBM [14]. The basic mechanism employed is mass damping through elastomers. We are currently building a vibration isolation unit of this ype and plan to add it to our existing system by simply placing it on the center stage of our current vibration solation unith is hoped that the two types of vibrational solation will be complimentary to each other, the spring amping being more eftective for lange-amplitude vibrations and the viton stainless-steel stack being more effective for the higher-frequency vibrations.

It will be advantageous in our work to be able to measure simultaneously a contour of constant electron density an the spatially resolved rate of decay of the surface wave function. In order to achieve this, some combination of constant-current scanning and distance-dependent-curren canning must be employed. We envision using a schem similar to the design used by Elrod et al. [15] for accomplishing this.

A sample-preparation chamber equipped with an airlock has been designed for the microscope and is being built. Th overall arrangement is to attach the sample-preparation chamber to the existing bell jar, separating the two by a gate valve. The airlock chamber is also isolated from the samplepreparation chamber by a gate valve and is located opposite the microscope chamber. A 30-inch-stroke translator (UHV Instruments Model 1000) which is used for moving samples among the three chambers is mounted on the opposite side of the airlock chamber. To introduce a new sample into the system, the airlock chamber is opened with both gate valves closed and the long-stroke translator fully retracted. The the sample can be placed on the translator, the airlock chamber sealed and evacuated, and the gate valve into the sample-preparation chamber opened to allow the translator and sample access. Similarly, if any of the procedures used in sample preparation involve pressures and reagents which are not desired in the microscope, the microscope can be isolated by means of the gate valve between the two chambers for the duration of the procedure and on will be sandard surface-analysis system.

The sample-preparation chamber and sample-loading airlock will greatly enhance the versatility of our instrumen. The sample-preparation chamber will provide a means of preparing well-characterized samples for microscope study. The airlock will allow the introduction of new substrates without the bakeout necessary when vacuum is broken. This addition should allow us to study many chemical systems with minimal downtime.

- Theoretical considerations

The theoretical component of our research is focused on assessing the changes in the tunneling current caused by variations in the size and structure of surface adsorbates. Our aim is to use theory as a means of qualitatively understanding how the physical properties of an adsorbate (e.g., ionization potential, electron affinity, atomic radius, etc.) might influence the tunneling current. Since the effects of such adsorbates can be assumed local, our calculations use clusters to model the surface and tip, and use $a b$ initio electronic structure techniques to obtain the cluster wave functions. The calculations are being performed in

association with the research group of W. A. Goddard III at Caltech. Such clusters have been used previously to model interactions of adsorbates with metal surfaces $[16,17]$.

Previous theoretical investigations of STM have bee carried out by a number of groups which have examined idealized surfaces [18-21] and reconstructed surfaces of metals and semiconductors [22,23], as well as the effects of single-atom adsorbates on the tunneling current density between two parallel plate electrodes [24] By using timedependent, first-order perturbation theory, the tunneling current between the surface and tip can be obtunneling approximately as $[22,23,25]$.

$I=\frac{2 \pi e}{\hbar} \sum_{\mu, \nu} f\left(E_{\mu}\right)\left[1-f\left(E_{\nu}+e V\right)\right]\left|T_{\mu \nu}\right|^{2} \delta\left(E_{\mu}-E_{\nu}\right)$,

where $f\left(E_{u}\right)$ is the Fermi function for the surface, $V$ is the applied voltage, and $T_{\text {wu }}$ is the electronic coupling matrix element between states $\psi_{\mu}$ and $\psi_{\nu}$ taken to be the surface and tip states, respectively. In the limits of low temperature and low applied voltage, Equation (1) reduces to $[22,23]$

$I=\frac{2 \pi e^{2} V}{\hbar} \sum_{\mu \nu}\left|T_{\mu \nu}\right|^{2} \delta\left(E_{\mu}-E_{\mathrm{F}}\right) \delta\left(E_{\nu}-E_{\mathrm{F}}\right)$.

is seen that the tunneling current is directly related to the size of the electronic matrix element $T_{\mu v}$. The full expression for $T_{\mu \nu}$ is $[26,27]$

$T_{\mu \nu}=\frac{\left\langle\psi_{\mu}\left|H_{\text {TOT }}\right| \psi_{\nu}\right\rangle-S_{\nu \mu}\left\langle\psi_{\nu}\left|H_{\text {TOT }}\right| \psi_{\nu}\right\rangle}{1-|S|^{2}}$.

Here $\psi_{\mu}$ and $\psi_{\nu}$ are assumed to be the full many-electron wave functions of the system with the "extra" electron

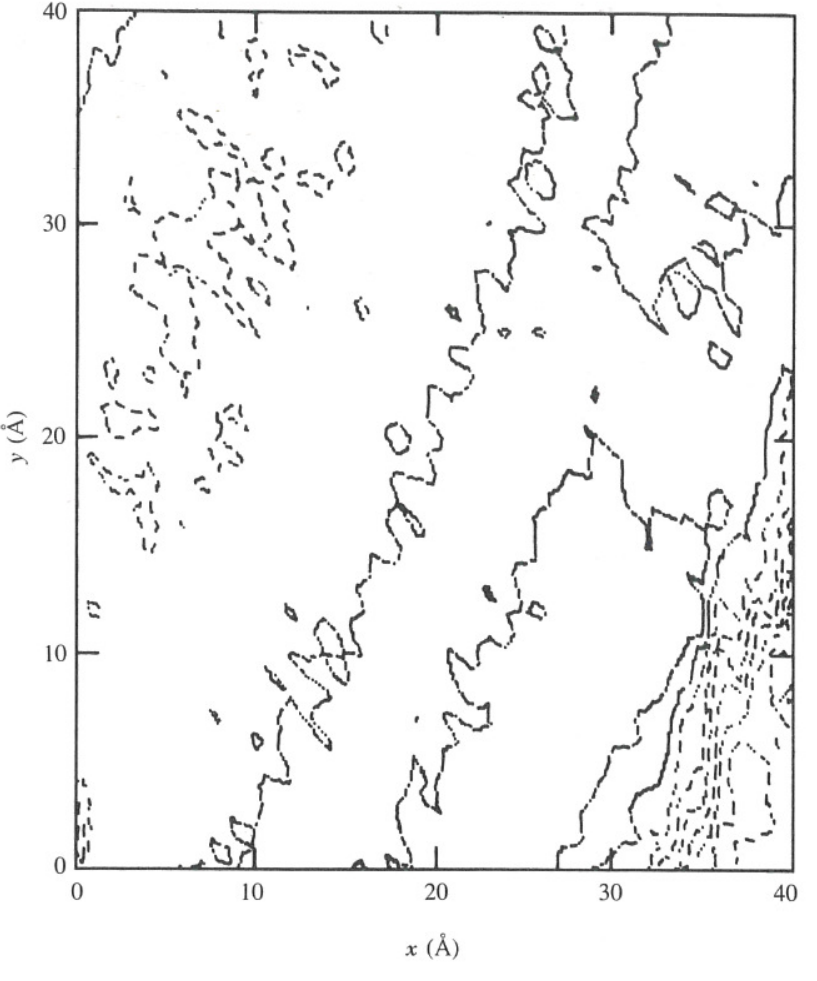

Figure 4

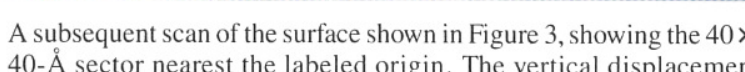
from highest to lowest contour is about $40 \AA$.

bound to the surface and tip respectively. In practice $\psi_{\mathrm{f}}$ and must be truncated. For example, when the

(a) the tip and surface a tran he tran in $\psi$ $\psi_{\mu} \psi_{\nu}$.

functions representing $\psi_{\mu}$ and $\psi_{v}$ are eigenfunctions
surface and tip potentials respectively, $T$ becomes

$$
T_{\mu \nu}=\frac{\left\langle\psi_{\mu}\left|V_{\text {tip }}\right| \psi_{\nu}\right\rangle-S_{v \mu}\left\langle\psi_{\nu}\left|V_{\text {tip }}\right| \psi_{\nu}\right\rangle}{1-\left|S_{\mu \nu}\right|^{2}} .
$$

Since $\psi_{u}$ and $\psi_{v}$ decay exponentially with distance from their respective surfaces, the second term in the numerator of (4) can be neglected, the denominator is essentially unity at large enough distances, and $T_{\mu \nu}$ becomes $T_{\mu \nu}=\left\langle\psi_{\mu}\left|V_{\text {tip }}\right| \psi_{\nu}\right\rangle$.

The quantity in Equation (5) was evaluated by Tersoff and Hamann [22,23] using the Bardeen expression [25] for the matrix element.

(3) In our studies we have chosen to use ab initio electronic structure techniques to examine $T_{\mu \nu}$. The main advantage in using these methods is that they should afford a reasonably accurate description of the adsorbate-surface interaction 


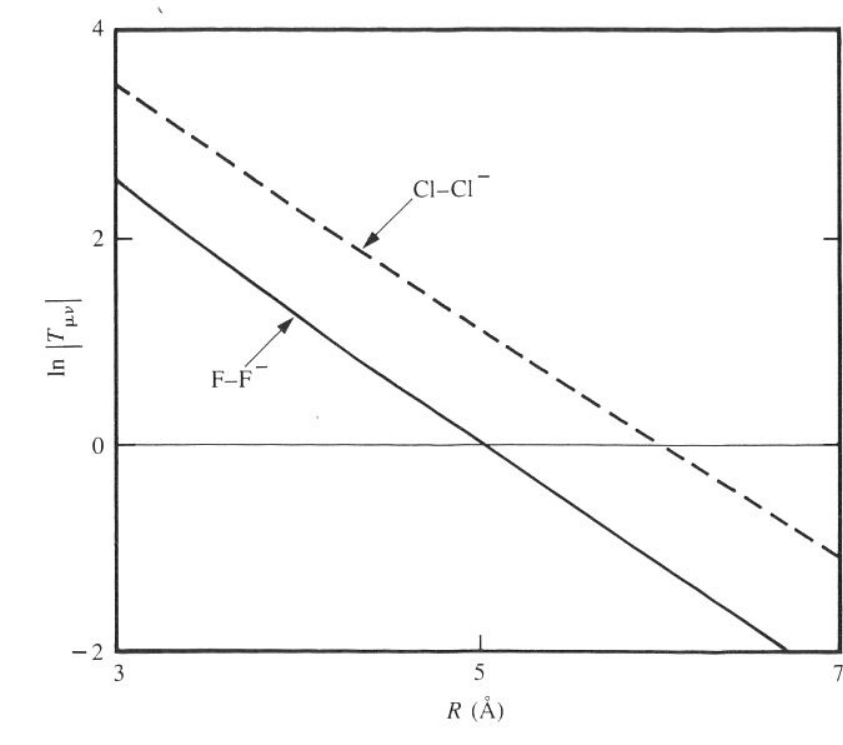

cluster description, the fractional gain or loss of charge can approach unity as the cluster size shrinks. The remaining electronic charge will respond to this by changing shape, which can, in principle, affect $T_{\mu}$

Our initial aim is to characterize the energy dependence and orbital shape dependence of $T_{\mu \nu}$ in relatively simple systems. Therefore, we have calculated $T_{w}$ as a function of distance for charge transfer between pairs of second-and third-row halide atoms, as well as pairs of alkaline-earth metals. From such results the effects of changes in ionizatio potential and atomic size can be examined, which should aid in interpreting results on more complex systems.

In Figure 5, results are shown for the tunneling matrix element as a function of distance in the systems $\mathrm{F}-\mathrm{F}^{-}$an $\mathrm{Cl}_{-} \mathrm{Cl}^{-}$. The wave functions were obtained at the HartreeFock level using standard basis sets [32]. The tunneling matrix element is seen to decay essentially exponentially with distance over the range of distances shown. Distances

Fignire up to $10 \AA$ were examined in the case of $\mathrm{F}-\mathrm{Cl}^{-}$(not shown) and the decay of $\ln \left|T_{\mu \nu}\right|$ was also found to be basically Plot of $\ln \left|T_{\mu \mu}\right|$ as a function of center-to-center separation distance
for the atomic pairs $\mathrm{F}-\mathrm{F}^{-}$and $\mathrm{Cl}-\mathrm{Cl}^{-}$. The values of $T_{\mu \nu}$ are in
millihartrees, the separation distances in $\AA$ A.

The truncation of $\psi_{\mu}$ and $\psi_{\nu}$ entails the limitation of the region of the surface and the tip to be cons dimensionally infinite slab calculations are currently unfeasible using $a b$ initio methods, due to the excessive integral evaluation time which would be required. $\psi$ and then become the many-electron wave functions describing then becoments and no further approximations are made the evaluation of Equation (3) for $T$ Such electronic structure techniques have been applied previously to the study of che-transfer reactions in aqueous solution [2830], and effient met for the evaluation of $T$, have been deverion $[28,31]$

There are certain disadvantages in using such techniques There are to chara cive 作 atom exp(-an) This lits the range of disp (cof arisons of the effects of a variety of adsorbates on $T$ (and therefore, hhe tunneling current, hese effects can be examined over distances where the basis set effects are not A secolem, and distances if desired.

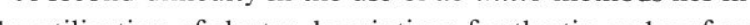

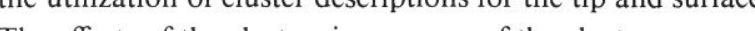
The efress of the electeric response or the clasters upon gain or loss of an elect

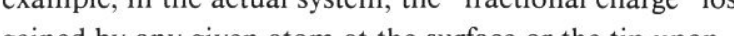
linear. Essentially identical results were obtained for $T$ beyond $4 \AA$ using wave functions for each center obtained i the absence of the second atom, thus indicating that the interaction between the centers is small at these distances, it should be.

Using Koopmann's theorem [33], an estimate of the ionization potential of a given orbital can be obtained from the Hartree-Fock orbital energy. For $\mathrm{F}^{-}$our results yield 4.9 eV and $\mathrm{for}^{-}$they yield $4.0 \mathrm{eV}$. On this basis it is expected that the decay of $T$ with distance should be somewhat greater when $\mathrm{F}^{-}$is involved than when it is not. The resuls of Figure 5 bear this out but it is apparent that the difference in decay rate is, in any event, rather small.

It can also be seen from Figure 5 that the size of $T$ can vary considerably at a given $R$ as a function of the atoms involved in the transfer. This affect difference in size of the $2 \mathrm{pF}$ orbitals and the $3 \mathrm{p} \mathrm{Cl}$ orbithe the the from the nucleus, th

Reve also been obtained for transfers in the systems $\mathrm{Be}-\mathrm{Be}^{+}$and $\mathrm{Mg}-\mathrm{Mg}^{+}$, and they are shown as functions of distance in Figure 6. The decay of $T_{\mu \nu}$ with seen to be essentially exponential with

The effects of orbital shape on the lateral displacement behavior of $T_{\mu \nu}$ are exam examined are show in Figre $7($ a). In Figure $7(b)$ the results labeled "Exact" are for calculations of $T_{\mu \nu}$ for $\Delta z=5.5 \AA$ "Estime" corm $-2 \mathrm{~A}$ to $+2 \mathrm{~A}$. The resulls labeled "Estinated correspond to an estimate of this variation based on the assumption that the decrease in $T_{\mu \nu}$ for nonzero $\delta$ is merely due to an increase in center-to-center separatio electron tayneris in line The adivios tributed to the nonspherical shape of the fluorine $2 p$ orbital.

Such results are, of course, preliminary but can serve as guides for more accurate surface and tip models. In

particular, we are currently using first-row transition metals as surface and tip models. Simple atomic and molecular adsorbates can then be bonded to the "surface" in order to examine the perturbations to $T_{w}$ caused by the adsorbate. The possible increase in electronic coupling between the initial and final states due to the presence of an intervening medium has been discussed previously [34,35].

There are several ways to assess the importance of effects such as the possible overadjustment of the remaining electronic cloud upon electron transfer on the calculated $T$ in the present model. First, using single-atom surface and tip models, results from calculations of $T_{\mu \nu}$ could be compare using systems having either frozen or relaxed cores. The present results used relaxed cores; that is, the cores were able to respond to the presence or absence of the extra electron a center. The frozen core results would give an indication of the extent of the effects of such relaxation on $T_{\mu}$

Another method of assessing these effects is to increase the cluster size. It is reasonable to assume that such problem will be greatest for the single-atom surface and tip model and will decrease as the cluster size increases. One area of interest which can be explored through the use of a varie of cluster sizes is the sensitivity of $T_{\mu \nu}$ to the tip size and shape. Models of proposed tip shăpes can be easily tested and the results compared with single-atom tips. While such tip-shape effects have been considered previously in a genera fashion $[10,19,22,23]$, apparently no molecular models have been examined yet.

- Systems to be studied

Our long-term objective for research using the STM is the study of systems of chemical interest. These studies will focus on such issues as the nature of the surface adsorbate interaction, chemical processes on surfaces, and the structure of the wave functions of adsorbates ranging in size from single atoms to macromolecules.

Benzene will probably be one of the first molecular species that we will study. Benzene chemisorbs to atomically flat clean metal surfaces by interaction of the ring $\pi^{*}$ orbitals with the appropriate metal surface orbitals. The $\mathrm{C}_{6}$ ring is generally parallel to the surface plane [36,37], but the position of the hydrogen atoms cannot be established from available spectroscopic data. Chemical studies suggest that the hydrogen atoms may lie further from the crystal plane than the $\mathrm{C}_{6}$ ring $[38,39]$. STM may provide a means of probing this structure. The various intermediates in benzen hydrogenation [40] may be investigated as well.

Another simple system which we will likely study with the STM is the coordination of pyridine to transition metal surfaces. Pyridine is believed to bind to $\mathrm{Pt}(111)$ and $(100)$ through the nitrogen atom on the basis of work-function

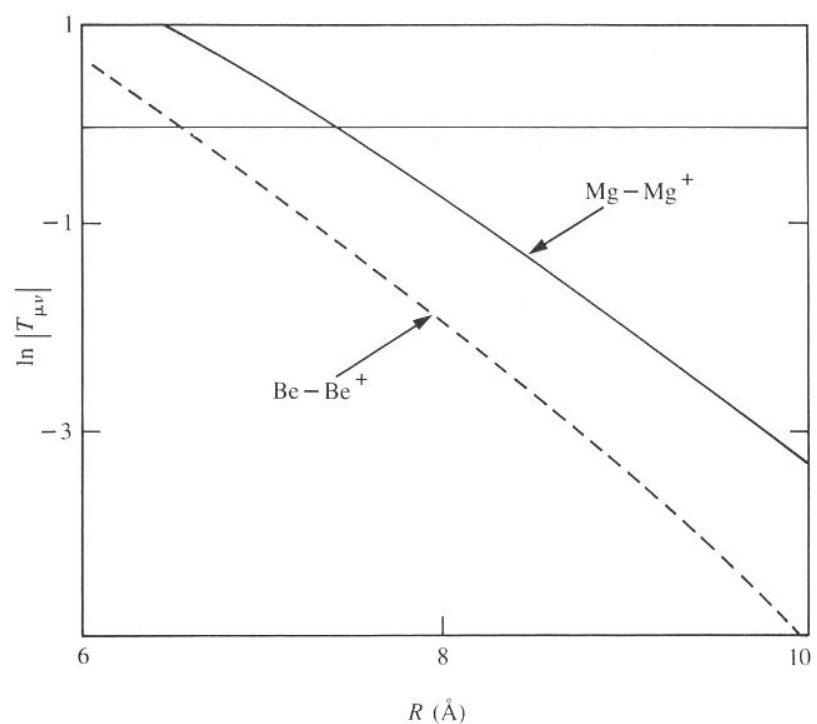

$R(\AA)$
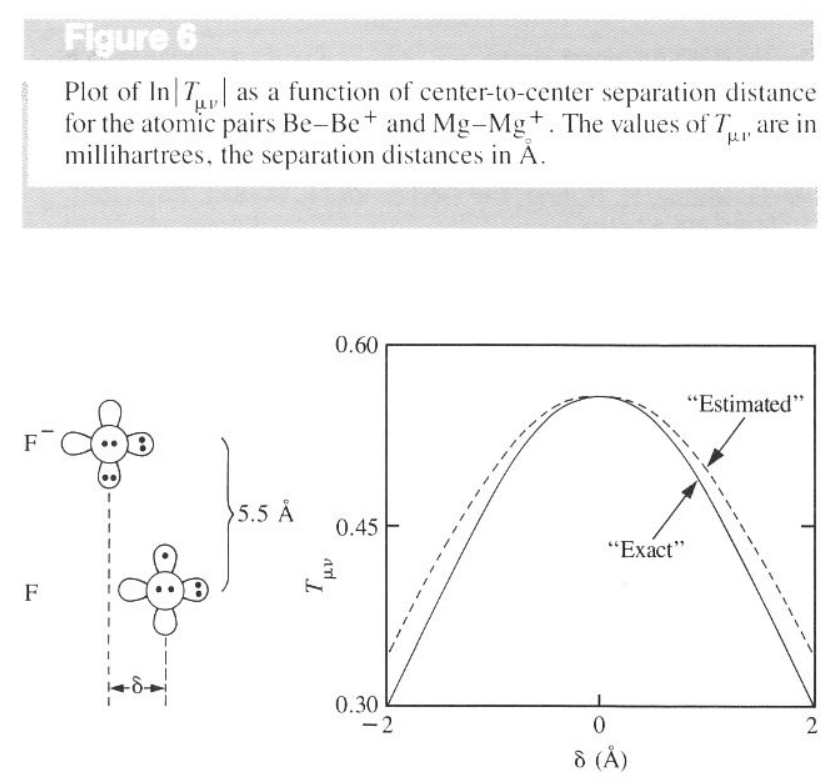

(a) Schematic description of the orientations between the two fluo-

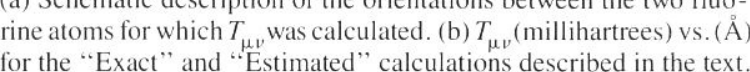

measurements [41,42]. Near-edge absorption studies have established that chemisorbed pyridine on $\operatorname{Pt}(111)$ is prependicular to the surface plane for coverages above 0.1 monolayers [43]. However, recent photoemission data indicate that either the nitrogen lone pair or the aromatic cloud, or both, may be involved in the bonding of pyridine to $\operatorname{Pd}(111)$ [44], $\operatorname{Ir}(111)$ [45], $\mathrm{Cu}(110)$ [46], and $\mathrm{Ag}(111)$ [47] The STM should yield useful information on the structure of surface-bound pyridine. 
Various porphyrins would appear to be ideal candidates for early studies of larger molecules. For example, the metalphthalycyanines are extremely stable, planar species of welknown molecular and electronic structure. They can be readily obtained containing metal atoms such as copper, and

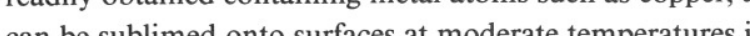

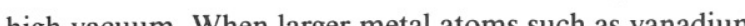
hich The in longer lies in the plone

\section{Acknowledgments}

The support of the Office of Naval Research (Contract No. N00014-84-K-0638), the Shell Company Foundation Inc. and the President's Fund of Caltech are gratefully acknowledged

\section{References and note}

1. Shuk Y. Tong, Phys. Today 37,50 (August 1984).
2. G. Binnig, H. Rohrer, Ch. Gerber, and E. Weibel, Appl. Phys. 2. G. Binnig, H. Rohrer, 3. G. Binnig, H. Rohrer, Ch. Gerber, and E. Weibel, Phys. Rev.

. G. Binnig and H. Rohrer, Surv. Phys. Acta 55, 726 (1982). 6. G. Binnig, H. Rohrer, Ch. Gerber, and E. Weibel, Phys. Rev. 7. A. Baratoff, G. Binnig, and H. Rohrer, J. Vac. Sci. Technol. B $\mathbf{1}$, 703 (1983).
8. G. Binnig. H. Rohrer, Ch. Gerber, and E. Weibel, Surf. Sci. 131, 8. G. Binnig, H. Rohrer, Ch. Gerber, and E. Weibel, Surf. Sci. 131,
$379(1983)$.

9. A. Baró, G. Binnig, H. Rohrer, Ch. Gerber, E. Stoll, A. Baratoff, and F. Salvan, Phys. Rev. Lett. 52,1304 (1984).
10. J. Pendry, Low Energy Electron Diffraction, Academic Press,

Carrol and J. May, Surf. Sci. 29, 60 and 85 (1972).

12. J. Simmons, J. Appl. Phys. 34, 1793 (1963).

14. C. Gerber, IBM Research Division, Zurich, Switzerland, private 15. S. Elrod, A. de Lozanne, and C. Quate, Appl. Phys. Lett. 45,
. 16. T. H. Upton and W. A. Goddard III, Phys. Rev. Lett. 42,472 (1979). 17. P. E. M. Siegbahn, M. R. A. Blomb). 18. N. Garcia, C. Ocal, and F. Flores, Phose 19. G. Binnig, N. Garcia, H. Rohrer, J. M. Soler, and F. Flore, Phys. Rev. B 30, 4816 (1984)

A Selloni, and P. Carnevali, J. Phys. C 17,

21. A. Baratoff, Europhys. Conf. Abstr. 76, 364 (1983). 22. J. Tersoff and D. R. Hamann, Phys. Rev. Lett. 50, 1998 (198

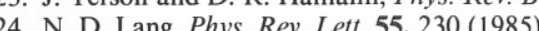

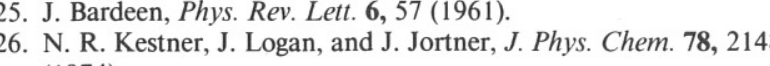
27. R. J. Cave, (197).

D. Th. Thesis, California Institute of Technology,

28. M. D. Newton, Int. J. Quantum Chem.: Quantum Chem. Symp. 29. J. Logan and M. D. Newton, J Chem. Phys. 78, 4086 (1983).
0. B. L. Tembe, H. L. Friedman, and M. D. Newton, J. Chem. Phys. 76, 1490 (1982). A. F. Voter, Ph.D. Thesis,
Pasadena, 1983.

(9s, 5p/3s, 2p) Huzinasercibe each F-atom were a standard set of negative ion functions $(\mathrm{s}$ exponent $=0.112$, pexponent $=$ 0.076 ) and a set of diffuse Rydberg functions (s exponent $=$ and $\mathrm{Mg}$, the Ne core in each exponent $=0.015$ ). . To describe effective potential of Rappe, Smedley, and Goddard [J. Phys. Chem. 85, 1662 (1981)]. The valence basis set for Cl comprised potential used, a set of $\mathrm{d}$ polarization functions (exponent $=0.6$ ) and a set of $p$ negative ion functions (exponent $=0.049$ ). For $\mathrm{Mg}$, a double zeta valence set appropriate to the SHC effective calculations was the standard (9s, 5p/3s, 2p) Huzinaga/Dunnin set.
3. Azabo and N. R. Ostlund, Modern Quantum Chemistry,

34. J. Halpern and L. E. Orgel, Discuss. Faraday Soc. 29, 32 (1960) 5. H. M. McConnell, J. Chem. Phys. 35, 508 (1951). 6. S. Lehwald, H. Ibach, and J. Demuth, Surf. Sci. 78,577 (1978) 38. M. Tsai and E. Muetterties, J. Amer. Chem Soc. 104, 2534

(1982).
39. M. Tsai and E. Muetterties, J. Phys. Chem. 86, 5067 (1982), 104, 2539 (1982)

41. J. Gland and G. Somorjai, Surf. Sci. 38, 157 (1973).
4. Gland and G. Somorjai, Adv. Coll. Interface Sci. 5, 205

A. Johnson, E. Muetterties, and J. Stöhr, J. Amer. Chem. So

45. F. Netzer and J. Mack, Chem. Phys. Lett. 95, 492 (1983).

46. B. Bandy D. Lloyd and N. Richardson, Surf. Sci 89).

47. J. Demuth, K. Christmann, and P. Sanda, Chem. Phys. Lett. 76 $201(1980)$

Received August 8, 1985; accepted for publication November

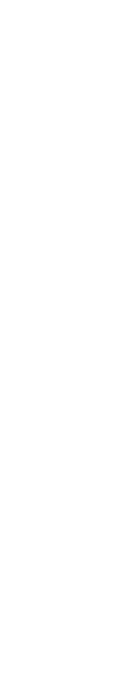

Paul West Aerojet Electro Systems Co., Surface Science member at Aerojet Electrosystems. Prior to joining Aerojet, he was a Postdoctoral Fellow at the California Institute of Technology, Pasadena, for the academic year $1983-84$. While at Caltech, he
designed and constructed the scanning tunneling microscope received his Ph.D. in physical chemistry from Wesleyan University, Middletown, Connecticut, in 1983. His research interests include operation and applicalon on laser and dye bra

John Kramar California Institute of Technology, Department of student in chemistry at Caltech. He is currently involved in the design and construction of a second-generation scanning tunneling microscope which will of a sea she-preparation chamber and

David V. Baxter McGill University, Montreal, Quebec, Canada. Postdoctoral Fellow doing research in the structural and electrical properties of noncrystalline metallic alloys using X-ray absorption, X-ray diffraction, and low-temperature transport measurements, as
well as collaborating with Dr. Robert Cave of the Caforita of Technology, Pasadena, on the theory of scanning tunneling microscopy. Prior to this, he was a Postdoctoral Fellow at the California Institute of Technology, where he received his Ph.D. in applied physics in
Physical Society.

Robert J. Cave California Institute of Technology, Department of Associate in Chemistry at Caltech; he joined the Baldeschwieler group in 1985. He received his Ph.D. at Caltech and from 1979 to research interests include solution electron trocctoral Fellow. His research interests include solution electron transfer theory and
theoretical investigations of atom-molecule collision dynamics.

John D. Baldeschwieler California Institute of Technology, Division of Chemistry and Chemical Engineering, Pasadena,
California 91125 . Dr. Baldeschwieler is Professor of Chemistry at Caltech. He received his Bachelor of Chemical Engineering degree his Ph.D. in physical folmistry from the University of California, Berkeley, in
1959 . The following University, Cambridge, Massachusetts, as Instructor and Assistan Professor of Chemistry. He moved to Stanford University in $1965 \mathrm{~s}$ as
Associate Professor and Professor of Chemistry. De. Baldechhwiler served as Deputy Director of the Office of Science and Technology in the Executive Office of the President from 1971 through 1973. He then joined the faculty of Caltech as Professor and Chairman of the for his work in developing nuclear magnetic double resonance techniques, ion cyclotron resonance spectroscopy, and perturbed angular correlation spectroscopy. His research interests also

Dr. Baldeschwieler has served on numerous national committess

and is a member of the American Philosophical Society and the
and 